Korean J. Math. 22 (2014), No. 1, pp. 71-84

http://dx.doi.org/10.11568/kjm.2014.22.1.71

\title{
WEAK CONVERGENCE FOR MULTIPLE STOCHASTIC INTEGRALS IN SKOROHOD SPACE
}

\author{
YOON TAE KIM
}

\begin{abstract}
By using the multidimensional normal approximation of functionals of Gaussian fields, we prove that functionals of Gaussian fields, as functions of $t$, converge weakly to a standard Brownian motion. As an application, we consider the convergence of the Stratonovich-type Riemann sums, as a function of $t$, of fractional Brownian motion with Hurst parameter $H=1 / 4$.
\end{abstract}

\section{Introduction}

Let $u(t, x)$ denote the solution to the stochastic heat equation

$$
u_{t}=\frac{1}{2} u_{x x}+\dot{W}(t, x), \text { with initial conditions } u(0, x)=0,
$$

where $\dot{W}$ is a space-time white noise on $[0, \infty) \times \mathbb{R}$. Then the solution $u(t, x)$ is given by

$$
u(t, x)=\int_{[0, t] \times \mathbb{R}} p(t-r, x-y) W(d r \times d y),
$$

Received January 8, 2014. Revised March 2, 2014. Accepted March 2, 2014.

2010 Mathematics Subject Classification: $60 \mathrm{H} 07$.

Key words and phrases: Malliavin calculus, Multiple stochastic integrals, Central limit theorem, Skorohod space, Gaussian process, fractional Brownian motion.

This research was supported by Basic Science Research Program through the National Research Foundation of Korea(NRF) funded by the Ministry of Education, Science and Technology (2012-012783).

(c) The Kangwon-Kyungki Mathematical Society, 2014.

This is an Open Access article distributed under the terms of the Creative commons Attribution Non-Commercial License (http://creativecommons.org/licenses/by -nc/3.0/) which permits unrestricted non-commercial use, distribution and reproduction in any medium, provided the original work is properly cited. 
where $p(t, x)$ is the heat kernel

$$
p(t, x)=\frac{1}{\sqrt{2 \pi t}} e^{-\frac{x^{2}}{2 t}} .
$$

For fixed $x \in \mathbb{R}$, let us set $F(t)=u(t, x)$. In [7], author studies the convergence of the sequence $J_{n}(t)$ of the following Stratonovich-type Riemann sums:

$$
J_{n}(t)=\sum_{j=1}^{[n t / 2]} F\left(\frac{2 j-1}{n}\right)\left[F\left(\frac{2 j}{n}\right)-F\left(\frac{2 j-2}{n}\right)\right] .
$$

In this paper, we extend the above problem (1) to the sequence $F_{n}$ belonging to the $q$ th Wiener chaos. For this, we use central limit theorems of multiple stochastic integrals, being the recent results proved by using the techniques of Malliavin calculus (see [4], [5] and [4]). The purpose of this paper is to study the convergence in distribution of a sequence of random functions of the form $F_{n}=I_{q}\left(f_{n}\right)$, where $I_{q}$ is the multiple stochastic integrals, by using Theorem 3.1. Here $I_{q}\left(f_{n}\right)(\omega)$ is a function in in the Skorohod space $D=D([0,1])$ and $I_{q}\left(f_{n}\right)(t)$ is a random variable.

As an application, we consider the convergence of the sequence $I_{n}(t)$ of the following Stratonovich-type Riemann sums:

$$
I_{n}(t)=\sum_{j=1}^{[n t / 2]} B^{H}\left(\frac{2 j-1}{n}\right)\left[B^{H}\left(\frac{2 j}{n}\right)-B^{H}\left(\frac{2 j-2}{n}\right)\right],
$$

where $B^{H}$ is a fractional Brownian motion with Hurst parameter $H=$ $1 / 4$. That is, let $B$ be a standard Brownian motion independent of $B^{H}$. The sequence $I_{n}$ weakly converges to $I$ in the Skorohod space $D$, where

(2) $I=\frac{1}{2}\left(B^{H}\right)^{2}-\frac{\sigma}{2} B, \sigma^{2}=2+\sum_{i=0}^{\infty}(-1)^{i}(2 \sqrt{i}-\sqrt{i-1}-\sqrt{i+1})^{2}$.

\section{Preliminaries}

In this section, we briefly review some basic facts about Malliavin calculus for Gaussian processes. For a more detailed reference, see [3]. Suppose that $\mathcal{H}$ is a real separable Hilbert space with scalar product denoted by $\langle\cdot, \cdot\rangle_{\mathcal{H}}$. Let $X=\{X(h), h \in \mathcal{H}\}$ be an isonormal Gaussian 
process, that is a centered Gaussian family of random variables such that $\mathbb{E}[X(h) X(g)]=\langle h, g\rangle_{\mathcal{H}}$. If $X=B^{H}$, then

$$
\mathbb{E}\left[B^{H}(t) B^{H}(s)\right]=\left\langle\mathbf{1}_{[0, s]}, \mathbf{1}_{[0, t]}\right\rangle_{\mathcal{H}}=\frac{1}{2}\left(t^{2 H}+s^{2 H}-|t-s|^{2 H}\right) .
$$

For every $q \geq 1$, let $\mathcal{H}_{q}$ be the $q$ th Wiener chaos of $X$, that is the closed linear subspace of $\mathbb{L}^{2}(\Omega)$ generated by $\left\{H_{q}(X(h)): h \in \mathcal{H},\|h\|_{\mathcal{H}}=1\right\}$, where $H_{q}$ is the $n$th Hermite polynomial. We define a linear isometric mapping $I_{q}: \mathcal{H}^{\odot q} \rightarrow \mathcal{H}_{q}$ by $I_{q}\left(h^{\otimes q}\right)=q ! H_{q}(X(h))$, where $\mathcal{H}^{\odot n}$ is the symmetric tensor product. The following duality formula holds

$$
\mathbb{E}\left[F I_{q}(h)\right]=\mathbb{E}\left[\left\langle D^{q} F, h\right\rangle_{\mathcal{H} \otimes q}\right],
$$

for any element $h \in \mathcal{H}^{\odot q}$ and any random variable $F \in \mathbb{D}^{q, 2}$. Here $\mathbb{D}^{q, 2}$ is the closure of the set of smooth random variables with respect to the norm

$$
\|F\|_{q, 2}^{2}=\mathbb{E}\left[F^{2}\right]+\sum_{k=1}^{q} \mathbb{E}\left[\left\|D^{k} F\right\|_{\mathcal{H} \otimes k}^{2}\right],
$$

where $D^{k}$ is the iterative Malliavin derivative. The linear isometric mapping $I_{q}$ satisfies $\left.I_{q}(f)=I_{q} \tilde{f}\right)$ and

$$
\mathbb{E}\left[I_{p}(f) I_{q}(g)\right]= \begin{cases}0 & \text { if } p \neq q \\ p !\langle\tilde{f}, \tilde{g}\rangle_{\mathcal{H}}^{\otimes p} & \text { if } p=q,\end{cases}
$$

where $\tilde{f}$ denotes the symmetrization of $f$.

If $f \in \mathcal{H}^{\odot p}$, the Malliavin derivative of the multiple stochastic integrals is given by

$$
D_{z} I_{q}\left(f_{q}\right)=q I_{q-1}\left(f_{q}(\cdot, z)\right) \text { for } z \in[0,1] .
$$

Let $\left\{e_{l}, l \geq 1\right\}$ be a complete orthonormal system in $\mathcal{H}$.

If $f \in \mathcal{H}^{\odot p}$ and $g \in \mathcal{H}^{\odot q}$, the contraction $f \otimes_{r} g, 1 \leq r \leq p \wedge q$, is the element of $\mathcal{H}^{\otimes(p+q-2 r)}$ defined by

$$
f \otimes_{r} g=\sum_{l_{1}, \cdots, l_{r}=1}^{\infty}\left\langle f, e_{l_{1}} \otimes \cdots \otimes e_{l_{r}}\right\rangle_{\mathcal{H}^{\otimes r}} \otimes\left\langle g, e_{l_{1}} \otimes \cdots \otimes e_{l_{r}}\right\rangle_{\mathcal{H}^{\otimes r}} .
$$

Notice that the tensor product $f \otimes g$ and the contraction $f \otimes_{r} g, 1 \leq r \leq$ $p \wedge q$, are not necessarily symmetric even though $f$ and $g$ are symmetric. We will denote their symmetrizations by $f \tilde{\otimes} g$ and $f \tilde{\otimes}_{r} g$, respectively. The following formula for the product of the multiple stochastic integrals will be frequently used to prove the main result in this paper (see [3]) 
Proposition 2.1. Let $f \in \mathcal{H}^{\odot p}$ and $g \in \mathcal{H}^{\odot q}$ be two symmetric functions. Then

$$
I_{p}(f) I_{q}(g)=\sum_{r=0}^{p \wedge q} r !\left(\begin{array}{l}
p \\
r
\end{array}\right)\left(\begin{array}{l}
q \\
r
\end{array}\right) I_{p+q-2 r}\left(f \otimes_{r} g\right) .
$$

\section{Main results}

Let us recall the following result in [2], which is a collection of some of the results contained in [4] and [6].

Theorem 3.1. Fix $d \geq 2$ and let $\Sigma=\left(\kappa_{i, j}\right)_{i, j=1, \ldots, d}$ be a $d \times d$ positive definite matrix. Fix integers $1 \leq q_{1} \leq \cdots \leq q_{d}$. For any $n \geq 1$ and $i=1, \ldots, d, f_{i}^{(n)} \in \mathcal{H}^{\odot q_{i}}$. Assume that

$$
F_{n}=\left(I_{q_{1}}\left(f_{1}^{(n)}\right), \ldots, I_{q_{d}}\left(f_{d}^{(n)}\right)\right), n \geq 1
$$

is such that

$$
\lim _{n \rightarrow \infty} \mathbb{E}\left[I_{q_{i}}\left(f_{i}^{(n)}\right) I_{q_{j}}\left(f_{j}^{(n)}\right)\right]=\kappa_{i, j}, \quad 1 \leq i, j \leq d .
$$

Then the followings are equivalent:

(i) For every $1 \leq i \leq d$, the sequence $\left\{I_{q_{i}}\left(f_{i}^{(n)}\right), n \geq 1\right\}$ converges to a normal distribution $\mathbb{N}\left(0, \kappa_{i, i}\right)$.

(ii) For every $1 \leq i \leq d, \lim _{n \rightarrow \infty} \mathbb{E}\left[\left(I_{q_{i}}\left(f_{i}^{(n)}\right)\right)^{4}\right]=3 \kappa_{i, i}^{2}$.

(iii) For every $1 \leq i \leq d$ and every $1 \leq r \leq q_{i}-1$,

$$
\lim _{n \rightarrow \infty}\left\|f_{i}^{(n)} \otimes_{r} f_{i}^{(n)}\right\|_{\mathcal{H}^{\otimes 2\left(q_{i}-r\right)}}=0 .
$$

(iv) The random vector $F_{n}$ converges in distribution to a $d$-dimensional Gaussian vector $\mathcal{N}_{d}(0, \Sigma)$.

We consider the Skorohod space $D=D([0,1])$.

Theorem 3.2. Suppose that for $t, s \in[0,1]$,

$$
\lim _{n \rightarrow \infty} \mathbb{E}\left[I_{q}\left(f_{n}(t)\right) I_{q}\left(f_{n}(s)\right)\right]=t \wedge s,
$$

where $f_{n}(t) \in \mathcal{H}^{\odot q}$ for each $t \in[0,1]$. For fixed $t \in[0,1]$, the sequence $\left\{I_{q}\left(f_{n}(t)\right), n \geq 1\right\}$ converges to a normal distribution $\mathcal{N}(0, t)$. We further 
assume that for $p=1, \ldots, q$,

$$
\begin{aligned}
& \left\|\left(f_{n}(t)-f_{n}\left(t_{1}\right)\right) \otimes_{q-p}\left(f_{n}(t)-f_{n}\left(t_{1}\right)\right)\right\|_{\mathcal{H} \odot 2 p} \\
& \times\left\|\left(f_{n}\left(t_{2}\right)-f_{n}(t)\right) \otimes_{q-p}\left(f_{n}(t)-f_{n}\left(t_{1}\right)\right)\right\|_{\mathcal{H} \odot 2 p} \\
\leq \quad & c_{p}\left[F\left(t_{2}\right)-F\left(t_{1}\right)\right]^{\vartheta}
\end{aligned}
$$

for $t_{1} \leq t \leq t_{2}$ and $n \geq 1$, where $\vartheta>1$, and $F$ is a nondecreasing, continuous function on $[0,1]$. Then we have

$$
I_{q}\left(f_{n}\right) \stackrel{\mathcal{D}}{\rightarrow} B
$$

where $B$ is a standard Brownian motion on $[0,1]$

Proof. We first show that the finite-dimensional distributions of $I_{q}\left(f_{n}\right)$ converge to those of $B$. For any $t_{1}, t_{2}, \ldots, t_{k} \in[0,1], k \geq 1$, let us set

$$
F_{n}=\left(I _ { q } \left(f_{n}\left(t_{1}\right), \ldots, I_{q}\left(f_{n}\left(t_{k}\right)\right) .\right.\right.
$$

By the assumption (8) and $\left\{I_{q}\left(f_{n}(t)\right), n \geq 1\right\} \stackrel{\mathcal{D}}{\rightarrow} \mathcal{N}(0, t)$, we obtain, from Theorem 3.1, that the random vector $F_{n}$ converges in distribution to a $k$ dimensional Gaussian vector $\mathcal{N}_{k}(0, \Sigma)$, where $\Sigma=\left(t_{i} \wedge t_{j}\right), i, j=1, \ldots, k$. For $t_{1} \leq t \leq t_{2}$ and $n \geq 1$, the formula for the product of the multiple stochastic integrals and (4) yield

$$
\begin{aligned}
& \mathbb{E}\left[\left(I_{q}\left(f_{n}(t)\right)-I_{q}\left(f_{n}\left(t_{1}\right)\right)\right)^{2}\left(I_{q}\left(f_{n}\left(t_{2}\right)\right)-I_{q}\left(f_{n}(t)\right)\right)^{2}\right] \\
= & \sum_{p=0}^{q}(p !)^{2}\left(\begin{array}{l}
q \\
p
\end{array}\right)^{4}(2 q-2 p) ! \times\left\langle\left(f_{n}(t)-f_{n}\left(t_{1}\right)\right) \otimes_{p}\left(f_{n}(t)-f_{n}\left(t_{1}\right)\right),\right. \\
& \left.f_{n}\left(t_{2}\right)-f_{n}(t)\right) \otimes_{p}\left(f_{n}\left(t_{2}\right)-f_{n}(t)\right\rangle_{\mathcal{H}^{\odot}(2 q-2 p)} .
\end{aligned}
$$

By using (9), the Cauchy-Schwartz inequality and $\left\|f \otimes_{p} f\right\|_{\mathcal{H}^{\odot}(2 q-2 p)}=$ $\left\|f \otimes_{q-p} f\right\|_{\mathcal{H} \odot 2 p}$, we get

$$
\begin{aligned}
& \mathbb{E}\left[\left(I_{q}\left(f_{n}(t)\right)-I_{q}\left(f_{n}\left(t_{1}\right)\right)\right)^{2}\left(I_{q}\left(f_{n}\left(t_{2}\right)\right)-I_{q}\left(f_{n}(t)\right)\right)^{2}\right] \\
\leq & \sum_{p=0}^{q}(p !)^{2}\left(\begin{array}{c}
q \\
p
\end{array}\right)^{4}(2 q-2 p) !\left\|\left(f_{n}(t)-f_{n}\left(t_{1}\right)\right) \otimes_{q-p}\left(f_{n}(t)-f_{n}\left(t_{1}\right)\right)\right\|_{\mathcal{H} \odot 2 p} \\
& \left.\times \| f_{n}\left(t_{2}\right)-f_{n}(t)\right) \otimes_{q-p}\left(f_{n}\left(t_{2}\right)-f_{n}(t) \|_{\mathcal{H} \odot 2 p}\right. \\
\leq & c_{q}\left[F\left(t_{2}\right)-F\left(t_{1}\right)\right]^{\vartheta},
\end{aligned}
$$

which implies that a sequence of cadlag processes $\left\{I_{q}\left(f_{n}(t)\right), t \in[0,1]\right\}$ is relatively compact in the Skorohod space $D([0,1])$. From Theorem 15.6 in [1], the result follows. 


\section{Applications}

By using our main result, we study the convergence of the sequence $I_{n}(t)$ of the following Stratonovich-type Riemann sums over a uniformly spaced time partition $t_{j}=j / n$ in the case when $H=1 / 4$.

$$
I_{n}(t)=\sum_{j=1}^{[n t / 2]} B^{H}\left(\frac{2 j-1}{n}\right)\left[B^{H}\left(\frac{2 j}{n}\right)-B^{H}\left(\frac{2 j-2}{n}\right)\right] .
$$

Let us set

$$
\begin{aligned}
F_{n}(t)= & \sum_{j=1}^{[n t / 2]}\left\{\left[B^{H}\left(\frac{2 j}{n}\right)-B^{H}\left(\frac{2 j-1}{n}\right)\right]^{2}\right. \\
& \left.-\left[B^{H}\left(\frac{2 j-1}{n}\right)-B^{H}\left(\frac{2 j-2}{n}\right)\right]^{2}\right\} .
\end{aligned}
$$

Theorem 4.1. Let $\sigma$ be as in (2) and $B$ be a standard Brownian motion. Then

$$
F_{n} \stackrel{\mathcal{D}}{\longrightarrow} B
$$

where the notation $\stackrel{\mathcal{D}}{\longrightarrow}$ denotes the convergence in distribution on the Skorohod space $D([0,1])$.

Proof. Using the formula for the product of the multiple stochastic integrals, we write

$$
\begin{aligned}
F_{n}(t)= & \sum_{j=1}^{[n t / 2]}\left\{I_{2}\left(\mathbf{1}_{\left[\frac{2 j-1}{n}, \frac{2 j}{n}\right]}^{\otimes 2}\right)-I_{2}\left(\mathbf{1}_{\left[\frac{2 j-2}{n}, \frac{2 j-1}{n}\right]}^{\otimes 2}\right)\right\} \\
& +\sum_{j=1}^{[n t / 2]}\left\{\left\|\mathbf{1}_{\left[\frac{2 j-1}{n}, \frac{2 j}{n}\right]}\right\|_{\mathcal{H}}^{2}-\left\|\mathbf{1}_{\left[\frac{2 j-2}{n}, \frac{2 j-1}{n}\right]}\right\|_{\mathcal{H}}^{2}\right\} \\
:= & F_{n, 1}(t)+F_{n, 2}(t) .
\end{aligned}
$$

If $n=2 k$ for $k=1,2, \ldots$, the sequence $\left\{F_{n, 1}(t)\right\}$ can be written as

$$
F_{n, 1}(t)=\sum_{j=1}^{[n t]}(-1)^{j} I_{2}\left(\mathbf{1}_{\left[\frac{j-1}{n}, \frac{j}{n}\right]}^{\otimes 2}\right) .
$$


If $n=2 k+1$ for $k=1,2, \ldots$, the sequence $\left\{F_{n, 1}(t)\right\}$ becomes

$$
F_{n, 1}^{*}(t)=\sum_{j=1}^{[(n-1) t]}(-1)^{j} I_{2}\left(\mathbf{1}_{\left[\frac{j-1}{n}, \frac{j}{n}\right]}^{\otimes 2}\right)
$$

When $n=2 k$ for $k=1,2, \ldots$, it is clear that

$$
\begin{aligned}
\mathbb{E}\left[F_{n, 1}(t)^{2}\right] & =2 \sum_{i, j=1}^{[n t]}(-1)^{i+j}\left\langle\mathbf{1}_{\left[\frac{i-1}{n}, \frac{i}{n}\right]}^{\otimes 2}, \mathbf{1}_{\left[\frac{j-1}{n}, \frac{j}{n}\right]}^{\otimes 2}\right\rangle_{\mathcal{H}}^{\otimes 2} \\
& =\frac{2[n t]}{n^{4 H}}+\sum_{r=1}^{[n t]-1}\left(\frac{[n t]-1-r}{n^{4 H}}\right)(-1)^{r} \rho(r)^{2}
\end{aligned}
$$

where $\rho(r)=2|r|^{2 H}-|r+1|^{2 H}-|r-1|^{2 H}$. Since $\sum_{r=1}^{\infty} \rho(r)^{2}<\infty$ for $H=1 / 4$, we obtain, by dominated convergence theorem, that the right-hand side in (14)

$$
\lim _{n \rightarrow \infty} \sum_{r=1}^{[n t]-1}\left(\frac{[n t]-1-r}{n^{4 H}}\right)(-1)^{r} \rho(r)^{2}=t \sum_{r=1}^{\infty}(-1)^{r} \rho(r)^{2}
$$

From this, we have

$$
\lim _{n \rightarrow \infty} \mathbb{E}\left[F_{n, 1}(t)^{2}\right]=t\left(2+\sum_{r=1}^{\infty}(-1)^{r} \rho(r)^{2}\right)
$$

Let us set $\sigma^{2}=2+\sum_{r=1}^{\infty}(-1)^{r} \rho(r)^{2}$. By using Theorem 3.1, we will prove that $\frac{1}{\sigma} F_{n, 1} \stackrel{\mathcal{D}}{\longrightarrow} B$, where $B$ is a standard Brownian motion. Fix $t \in[0,1]$. The Malliavin derivative of $F_{n, 1}(t)$ is given by

$$
D F_{n, 1}(t)=2 \sum_{j=1}^{[n t]}(-1)^{j} I_{1}\left(\mathbf{1}_{\left[\frac{j-1}{n}, \frac{j}{n}\right]}\right) \mathbf{1}_{\left[\frac{j-1}{n}, \frac{j}{n}\right]} .
$$


Hence, by the formula for the product of the multiple stochastic integrals, we have that for $0<t \leq 1$,

$$
\begin{aligned}
& \mathbb{E}\left[\left(\left\|D F_{n, 1}(t)\right\|_{\mathcal{H}}^{2}-2 t \sigma^{2}\right)^{2}\right] \\
= & 16 \sum_{i, j, k, l=1}^{[n t]}(-1)^{i+j}(-1)^{k+l}\left\langle\mathbf{1}_{\left[\frac{i-1}{n}, \frac{i}{n}\right]}, \mathbf{1}_{\left[\frac{j-1}{n}, \frac{j}{n}\right]}\right\rangle_{\mathcal{H}}\left\langle\mathbf{1}_{\left[\frac{k-1}{n}, \frac{k}{n}\right]}, \mathbf{1}_{\left[\frac{l-1}{n}, \frac{l}{n}\right]}\right\rangle_{\mathcal{H}} \\
& \times \mathbb{E}\left[I_{2}\left(\mathbf{1}_{\left[\frac{i-1}{n}, \frac{i}{n}\right]} \otimes \mathbf{1}_{\left[\frac{j-1}{n}, \frac{j}{n}\right]}\right) I_{2}\left(\mathbf{1}_{\left[\frac{k-1}{n}, \frac{k}{n}\right]} \otimes \mathbf{1}_{\left[\frac{l-1}{n}, \frac{l}{n}\right]}\right)\right] \\
& +16 \sum_{i, j, k, l=1}^{[n t]}(-1)^{i+j}(-1)^{k+l}\left\langle\mathbf{1}_{\left[\frac{i-1}{n}, \frac{i}{n}\right]}, \mathbf{1}_{\left[\frac{j-1}{n}, \frac{j}{n}\right]}\right\rangle_{\mathcal{H}}^{2}\left\langle\mathbf{1}_{\left[\frac{k-1}{n}, \frac{k}{n}\right]}, \mathbf{1}_{\left[\frac{l-1}{n}, \frac{l}{n}\right]}\right\rangle_{\mathcal{H}}^{2} \\
& -16 t \sigma^{2} \sum_{i, j=1}^{[n t]}(-1)^{i+j}\left\langle\mathbf{1}_{\left[\frac{i-1}{n}, \frac{i}{n}\right]}, \mathbf{1}_{\left[\frac{j-1}{n}, \frac{j}{n}\right]}\right\rangle_{\mathcal{H}}^{2}+4 t^{2} \sigma^{2} \\
:= & A_{n}(t)+B_{n}(t)+C_{n}(t)+4 t^{2} \sigma^{2} .
\end{aligned}
$$

The equation (4) yields

$$
\begin{aligned}
A_{n}(t)= & 16 \sum_{i, j, k, l=1}^{[n t]}(-1)^{i+j}(-1)^{k+l}\left\langle\mathbf{1}_{\left[\frac{i-1}{n}, \frac{i}{n}\right]}, \mathbf{1}_{\left[\frac{j-1}{n}, \frac{j}{n}\right]}\right\rangle_{\mathcal{H}} \\
& \times\left\langle\mathbf{1}_{\left[\frac{k-1}{n}, \frac{k}{n}\right]}, \mathbf{1}_{\left[\frac{l-1}{n}, \frac{l}{n}\right]}\right\rangle_{\mathcal{H}}\left\langle\mathbf{1}_{\left[\frac{i-1}{n}, \frac{i}{n}\right]}, \mathbf{1}_{\left[\frac{k-1}{n}, \frac{k}{n}\right]}\right\rangle_{\mathcal{H}}\left\langle\mathbf{1}_{\left[\frac{j-1}{n}, \frac{j}{n}\right]}, \mathbf{1}_{\left[\frac{l-1}{n}, \frac{l}{n}\right]}\right\rangle_{\mathcal{H}} \\
& +16 \sum_{i, j, k, l=1}^{[n t]}(-1)^{i+j}(-1)^{k+l}\left\langle\mathbf{1}_{\left[\frac{i-1}{n}, \frac{i}{n}\right]}, \mathbf{1}_{\left[\frac{j-1}{n}, \frac{j}{n}\right]}\right\rangle_{\mathcal{H}} \\
& \times\left\langle\mathbf{1}_{\left[\frac{k-1}{n}, \frac{k}{n}\right]}, \mathbf{1}_{\left[\frac{l-1}{n}, \frac{l}{n}\right]}\right\rangle_{\mathcal{H}}\left\langle\mathbf{1}_{\left[\frac{i-1}{n}, \frac{i}{n}\right]}, \mathbf{1}_{\left[\frac{l-1}{n}, \frac{l}{n}\right]}\right\rangle_{\mathcal{H}}\left\langle\mathbf{1}_{\left[\frac{j-1}{n}, \frac{j}{n}\right]}, \mathbf{1}_{\left[\frac{k-1}{n}, \frac{k}{n}\right]}\right\rangle_{\mathcal{H}} \\
:= & A_{n, 1}(t)+A_{n, 2}(t) .
\end{aligned}
$$

For the first sum, we decompose the sum $\sum_{i, j, k, l=1}^{[n t]}$ as follows:

$$
\begin{aligned}
& \sum_{i=j=k=l}+(\underbrace{\left.\sum_{i=j=k}+\cdots+\sum_{\substack{j=k=l \\
i \neq j}}\right)}_{\text {four sums }}+\left(\sum_{\substack{i=j, k=l \\
k \neq i}}+\sum_{\substack{i=k, j=l \\
i \neq j}}+\sum_{\substack{i=l, j=k \\
i \neq j}}\right) \\
& +(\underbrace{\left.\sum_{\substack{i=j, k \neq i \\
k \neq l, l \neq i}}+\cdots+\sum_{\substack{k \neq l, k \neq i \\
k \neq j, i \neq j}}\right)}_{\text {six sums }}+\sum_{i, j, k, l \text { are all different }} \cdot
\end{aligned}
$$


For the convergence of the above sums, we need to estimate

$$
\begin{aligned}
& \sqrt{n}\left|\left\langle\mathbf{1}_{\left[\frac{i-1}{n}, \frac{i}{n}\right]}, \mathbf{1}_{\left[\frac{j-1}{n}, \frac{j}{n}\right]}\right\rangle_{\mathcal{H}}\right| \\
= & \frac{1}{2}|(\sqrt{|j-i|}-\sqrt{|i-j-1|})-(\sqrt{|j-i+1|}-\sqrt{|i-j|})| .
\end{aligned}
$$

Let $f(x)=\sqrt{x+1}-\sqrt{x}$. Fix $j-i \geq 2$, the mean value theorem shows that for some $\lambda \in[0,1]$

$$
\begin{aligned}
& \sqrt{n}\left|\left\langle\mathbf{1}_{\left[\frac{i-1}{n}, \frac{i}{n}\right]}, \mathbf{1}_{\left[\frac{j-1}{n}, \frac{j}{n}\right]}\right\rangle_{\mathbb{H}}\right| \\
= & \frac{1}{2}\left|f^{\prime}(j-i-\lambda)\right| \leq \frac{1}{2 \sqrt{2}(j-i) \sqrt{j-i}} .
\end{aligned}
$$

In the case when $j-i=1$, since $2-\sqrt{2} \leq 1 / \sqrt{2}$, the inequality (18) holds. Hence

$$
\leq \frac{\left|\left\langle\mathbf{1}_{\left[\frac{i-1}{n}, \frac{i}{n}\right]}, \mathbf{1}_{\left[\frac{j-1}{n}, \frac{j}{n}\right]}\right\rangle_{\mathcal{H}}\right|}{2 \sqrt{2} \sqrt{n}|j-i| \sqrt{|j-i|}} \mathbf{1}_{\{|j-i| \geq 1\}}+\frac{1}{\sqrt{n}} \mathbf{1}_{\{j=i\}} .
$$

Let us set

$$
\begin{aligned}
B_{n}(i, j, k, l)= & \left\langle\mathbf{1}_{\left[\frac{i-1}{n}, \frac{i}{n}\right]}, \mathbf{1}_{\left[\frac{j-1}{n}, \frac{j}{n}\right]}\right\rangle_{\mathcal{H}}\left\langle\mathbf{1}_{\left[\frac{k-1}{n}, \frac{k}{n}\right]}, \mathbf{1}_{\left[\frac{l-1}{n}, \frac{l}{n}\right]}\right\rangle_{\mathcal{H}} \\
& \times\left\langle\mathbf{1}_{\left[\frac{i-1}{n}, \frac{i}{n}\right]}, \mathbf{1}_{\left[\frac{k-1}{n}, \frac{k}{n}\right]}\right\rangle_{\mathcal{H}}\left\langle\mathbf{1}_{\left[\frac{j-1}{n}, \frac{j}{n}\right]}, \mathbf{1}_{\left[\frac{l-1}{n}, \frac{l}{n}\right]}\right\rangle_{\mathcal{H}} .
\end{aligned}
$$

For the first sum, it follows from (19) that for each $0<t \leq 1$

$$
\left|16 \sum_{i=j=k=l}^{[n t]}(-1)^{i+j}(-1)^{k+l} B_{n}(i, j, k, l)\right| \leq c \frac{[n t]}{n^{2}} \rightarrow 0 .
$$

For the second sum, we have, from (19), that

$$
\left|16 \sum_{\mathcal{A}_{i j k l, 1}^{n}}(-1)^{i+j}(-1)^{k+l} B_{n}(i, j, k, l)\right| \leq \frac{c}{n} \sum_{l=1}^{n} \frac{1}{l^{3}} \rightarrow 0,
$$

where $\mathcal{A}_{i j k l, 1}^{n}=\{1 \leq i, j, k, l \leq[n t]: i=j=k, l \neq i\}$. For the third, the fourth and the fifth sums, we can proceed analogously and we obtain $C / n$ for bound. For the sixth sum, we have

$$
\left|16 \sum_{\mathcal{A}_{i j k l, 2}^{n}}(-1)^{i+j}(-1)^{k+l} B_{n}(i, j, k, l)\right| \leq \frac{c}{n} \sum_{k=1}^{n} \frac{1}{k^{3}} \rightarrow 0,
$$


where $\mathcal{A}_{i j k l, 2}^{n}=\{1 \leq i, j, k, l \leq[n t]: i=j, k=l, k \neq i\}$ For the seventh and the eighth sums, we can proceed analogously and we obtain $C / n$ for bound. For the ninth sum, we have

$$
\begin{aligned}
& \left|16 \sum_{\mathcal{A}_{i j k l, 3}^{n}}(-1)^{i+j}(-1)^{k+l} B_{n}(i, j, k, l)\right| \\
\leq & \frac{c}{n^{2}} \sum_{\substack{k \neq i \\
k \neq l, l \neq i}} \frac{1}{|k-l|^{3 / 2}} \frac{1}{|k-i|^{3 / 2}} \frac{1}{|l-i|^{3 / 2}},
\end{aligned}
$$

where $\mathcal{A}_{i j k l, 3}^{n}=\{1 \leq i, j, k, l \leq[n t]: i=j, k \neq i, k \neq l, l \neq i\}$ We can decompose the sum $\sum_{\substack{k \neq i \\ k \neq l, l \neq i}}$ into six sums

$$
\sum_{k>l>i}+\sum_{k>i>l}+\cdots \sum_{i>k>l}
$$

Since $k-i>k-l$, we have

$$
\begin{aligned}
& \frac{c}{n^{2}} \sum_{n \geq k>l>i} \frac{1}{|k-l|^{3 / 2}} \frac{1}{|k-i|^{3 / 2}} \frac{1}{|l-i|^{3 / 2}} \\
\leq & \frac{c}{n} \sum_{l=1}^{n} \frac{1}{l^{3}} \sum_{i=1}^{n} \frac{1}{i^{3 / 2}} \rightarrow 0 .
\end{aligned}
$$

These arguments give the same bound for the other sums in (21). By using the same way as for the ninth sum, we can show that the other sums in the fourth part in (16) have the bound $C / n$. For the last sum in (16), we can decompose the sum $\sum_{i, j, k, l}$ are all different into 4 ! sums

$$
\sum_{k>l>i>j}+\sum_{k>l>j>i}+\cdots
$$

For the first sum, we have

$$
\begin{aligned}
& \frac{c}{n^{2}} \sum_{k>l>i>j} \frac{1}{|i-j|^{3 / 2}} \frac{1}{|k-l|^{3 / 2}} \frac{1}{|k-i|^{3 / 2}} \frac{1}{|l-i|^{3 / 2}} \\
\leq & \frac{c}{n} \sum_{l=1}^{n} \frac{1}{l^{3}} \sum_{i=1}^{n} \frac{1}{i^{3 / 2}} \sum_{i=1}^{n} \frac{1}{j^{3 / 2}} \rightarrow 0 .
\end{aligned}
$$

By using the same as for the first sum in (22), we can show that the same bound also holds for the other sums in (22). By combining the above results, we can show that $A_{n, 1}(t) \rightarrow 0$. By using the same arguments as 
for $A_{n, 1}(t)$, we have that $A_{n, 2}(t) \rightarrow 0$, and hence $A_{n}(t) \rightarrow 0$. From (15), we can show that $B_{n}(t) \rightarrow 4 t^{2} \sigma^{4}$ and $C_{n}(t) \rightarrow 8 t^{2} \sigma^{4}$. Hence we have that $t \in[0,1]$

$$
D F_{n, 1}(t) \rightarrow 2 t \sigma^{2} \text { in } L^{2}
$$

When $n=2 k+1$ for $k=1,2, \ldots$, it is obvious that (23) also holds. It is immediately check that the sequence $\left\{F_{n, 2}(t)\right\}$ converges to zero as $n$ tends to infinity. The result in [4] proves that For every $t \in[0,1]$,

$$
\frac{1}{\sigma} F_{n, 1}(t) \stackrel{\mathcal{D}}{\longrightarrow} B(t)
$$

where $B$ is a standard Brownian motion. If we show that

$$
\lim _{n \rightarrow \infty} \mathbb{E}\left[F_{n, 1}(t) F_{n, 1}(s)\right]=(t \wedge s) \sigma^{2},
$$

we obtain, from Theorem 3.1, that for $t_{1}, t_{2}, \ldots, t_{l} \in[0,1], l \geq 1$,

$$
\frac{1}{\sigma}\left(F_{n, 1}\left(t_{1}\right), \ldots, F_{n, 1}\left(t_{l}\right)\right) \stackrel{\mathcal{D}}{\longrightarrow}\left(B\left(t_{1}\right), \ldots, B\left(t_{l}\right)\right) .
$$

Indeed, for $s \leq t$, it is clear that

$$
\begin{aligned}
\mathbb{E}\left[F_{n, 1}(t) F_{n, 1}(s)\right]= & \frac{2[n s]}{n}+\sum_{r=1}^{[n s]-1}\left(\frac{[n s]-1-r}{n}\right)(-1)^{r} \rho(r)^{2} \\
& \rightarrow s \sigma^{2} .
\end{aligned}
$$

For $s \leq t$, we estimate, from (19),

$$
\begin{aligned}
& \left\|\left(F_{n, 1}(t)-F_{n, 1}(s)\right) \otimes_{1}\left(F_{n, 1}(t)-F_{n, 1}(s)\right)\right\|_{\mathcal{H} \odot 2}^{2} \\
\leq & \left(\sum_{r=1}^{\infty} \rho(r)^{2}\right)^{2}\left(\frac{[n t]-[n s]}{n}\right)^{2} .
\end{aligned}
$$

By a similar estimate as for (28), we get

$$
\begin{aligned}
& \left\|\left(F_{n, 1}(t)-F_{n, 1}(s)\right) \otimes\left(F_{n, 1}(t)-F_{n, 1}(s)\right)\right\|_{\mathcal{H} \odot 2}^{2} \\
\leq & \left(\sum_{r=1}^{\infty} \rho(r)^{2}\right)^{2}\left(\frac{[n t]-[n s]}{n}\right)^{2} .
\end{aligned}
$$

The estimates (28) and (29) prove that (9) holds. Hence by Theorem 3.2 , the result (13) follows in the case when $n=2 k$. It is clear that the result (13) follows in the case when $n=2 k+1$. 
Lemma 4.2. Let $F_{n}$ be defined as in (12). Then

$$
\left(B^{H}, F_{n}\right) \stackrel{\mathcal{D}}{\longrightarrow}\left(B^{H}, B\right),
$$

where $B$ is a standard Brownian motion independent of $B^{H}$.

Proof. Fix $0<t_{1}<\cdots<t_{l}$. Then the random vector

$$
\left(\left(B^{H}\left(t_{i}\right)\right)_{1 \leq i \leq l},\left(I\left(\mathbf{1}_{\left[\frac{j-1}{n}, \frac{j}{n}\right]}\right)_{1 \leq j \leq n}\right)\right.
$$

is a $l+n$-dimensional normal distribution with mean vector 0 and covariance matrix

$$
\Sigma=\left(\begin{array}{ll}
\Sigma_{11} & \Sigma_{12} \\
\Sigma_{21} & \Sigma_{22}
\end{array}\right)
$$

where $\Sigma_{11}=\left(\mathbb{E}\left[B^{H}\left(t_{i}\right) B^{H}\left(t_{j}\right)\right]\right)_{1 \leq i, j \leq l}, \Sigma_{12}=\left(\mathbb{E}\left[B^{H}\left(t_{i}\right) I\left(\mathbf{1}_{\left[\frac{j-1}{n}, \frac{j}{n}\right]}\right)\right]\right)_{\substack{1 \leq i \leq l \\ 1 \leq j \leq n}}$ and $\Sigma_{22}=(\rho(i-j))_{1 \leq i, j \leq n}$. Then $\left(I\left(\mathbf{1}_{\left[\frac{j-1}{n}, \frac{j}{n}\right]}\right)\right)_{1 \leq j \leq n}-\Sigma_{21} \Sigma_{11}^{-1}\left(B^{H}\left(t_{i}\right)\right)_{1 \leq i \leq l}$ and $\left(B^{H}\left(t_{i}\right)\right)_{1 \leq i \leq l}$ are independent. Using the formula for the product of the multiple stochastic integrals, we write

$$
F_{n}(t)=\sum_{j=1}^{[n t]}(-1)^{j}\left(I_{1}\left(\mathbf{1}_{\left[\frac{j-1}{n}, \frac{j}{n}\right]}\right)\right)^{2}-(-1)^{j} \frac{[n t]}{n} .
$$

Define

$$
\begin{aligned}
F_{n}^{*}(t)= & F_{n}(t)-2 \sum_{j=1}^{[n t]}(-1)^{j} I_{1}\left(\mathbf{1}_{\left[\frac{j-1}{n}, \frac{j}{n}\right]}\right) \sum_{k=1}^{l} a_{j k} B^{H}\left(t_{k}\right) \\
& +\sum_{j=1}^{[n t]}(-1)^{j}\left(\sum_{k=1}^{l} a_{j k} B^{H}\left(t_{k}\right)\right)^{2},
\end{aligned}
$$

where $\Sigma_{21} \Sigma_{11}^{-1}=\left(a_{j k}\right)_{1 \leq \leq n, 1 \leq k \leq l}$. Note that

$$
\begin{aligned}
& \sup _{t \in[0,1]} \sum_{i=1}^{\infty} \mid \mathbb{E}\left[B^{H}(t)\left(B^{H}\left(\frac{i}{n}\right)-B^{H}\left(\frac{i-1}{n}\right)\right] \mid<\infty,\right. \\
& \sup _{t \in[0,1]} \mid \mathbb{E}\left[B^{H}(t)\left(B^{H}\left(\frac{i}{n}\right)-B^{H}\left(\frac{i-1}{n}\right)\right] \mid \leq \frac{1}{\sqrt{n}} .\right.
\end{aligned}
$$


Then, from (31) and (32), we get

$$
\begin{aligned}
& \mathbb{E}\left[\sup _{0 \leq t \leq 1}\left|F_{n}(t)-F_{n}^{*}(t)\right|\right] \\
\leq & \frac{c}{n^{1 / 4}}+\frac{c}{\sqrt{n}} \sum_{j=1}^{n} \sum_{k=1}^{l}\left|\mathbb{E}\left[I_{1}\left(\mathbf{1}_{\left[\frac{j-1}{n}, \frac{j}{n}\right]}\right) B^{H}\left(t_{k}\right)\right]\right| \rightarrow 0 \text { as } n \rightarrow \infty .
\end{aligned}
$$

Thus it follows from (13) and (33) that

$\left(\left(B^{H}\left(t_{i}\right)\right)_{1 \leq i \leq l}, F_{n}^{*}\left(s_{1}\right), \ldots, F_{n}^{*}\left(s_{k}\right)\right) \stackrel{\mathcal{D}}{\longrightarrow}\left(\left(B^{H}\left(t_{i}\right)\right)_{1 \leq i \leq l}, B\left(s_{1}\right), \ldots, B\left(s_{k}\right)\right)$.

Since $\left(B^{H}\left(t_{i}\right)\right)_{1 \leq i \leq l}$ and $F_{n}^{*}$ are independent, and the sequence $\left(B^{H}, F_{n}\right)$ is relatively compact, the proof is complete.

Theorem 4.3. Let $\sigma$ be as in (2) and and $B$ be a standard Brownian motion. Then

$$
I_{n} \stackrel{\mathcal{L}}{\longrightarrow} \frac{1}{2}\left(B^{H}\right)^{2}-\frac{\sigma}{2} B
$$

where $B$ is a standard Brownian motion independent of $B^{H}$.

Proof. Using $a^{2}-b^{2}=(a+b)(a-b)$, the sequence $\left\{F_{n}\right\}$ in (12) can be written as

$$
F_{n}(t)=-2 I_{n}(t)+B^{H}\left(\frac{2[n t / 2]}{n}\right)^{2}-B^{H}(t)^{2}+B^{H}(t)^{2} .
$$

From (35), we write

$$
I_{n}(t)=\frac{1}{2} B^{H}(t)^{2}+\left[B^{H}\left(\frac{2[n t / 2]}{n}\right)^{2}-B^{H}(t)^{2}\right]-F_{n}(t) .
$$

It is clear that

$$
\mathbb{E}\left[\sup _{0 \leq t \leq 1}\left|B^{H}\left(\frac{2[n t / 2]}{n}\right)^{2}-B^{H}(t)^{2}\right|\right] \rightarrow 0 .
$$

By Lemma 4.2, the proof is complete.

\section{References}

[1] Billingsley. P. , Converges of probability measures, John Wiley \& Sons, Inc., 1968.

[2] Nourdin, I., Peccati, G. and Réveillac, A., Multivariate normal approximnation using Stein's method and Malliavin calculus, Ann. Inst. Henri Poincaré Probab. Stat. 46 (1) (2010), 45-58.

[3] D. Nualart, Malliavin calculus and related topics, 2nd Ed. Springer. 2006. 
[4] Nualart, D. and Ortiz-Latorre, S., Central limit theorems for multiple stochastic integrals and Malliavin calculus, Stochastic Process. Appl. 118 (2008), 614-628.

[5] Nualart, D. and Peccati, G., Central limit theorems for sequences of multiple stochastic integrals, Ann. Probab. 33 (1) (2005), 173-193.

[6] Peccati, G. and Tudor, C.A. (2005)., Gaussian limits for vector-valued multiple stochastic integrals. In: Séminaire de Probabilités XXXVIII. Lecture Notes in Math., 1857, 247-262. Springer, Berlin.

[7] Swanson, J. Variations of the solution to a stochastic heat equation, Ann. Probab. 35 (6) (2007), 2122-2159.

Yoon Tae Kim

Department of Finance and Information Statistics

Hallym University

Chunchon 200-702, Korea

E-mail: ytkim@hallym.ac.kr 